\title{
Chikungunya Arbovirus in Bangladesh: An Epidemic with Medical Entomology
}

\author{
Hassan $\mathrm{MM}^{1 *}$, Jhora $\mathrm{ST}^{2}$, Ahmed $\mathrm{M}^{3}$, Hayatun Nabi $\mathrm{M}^{4}$, Huq $\mathrm{N}^{5}$, Rahman $\mathrm{S}^{6}$, \\ Sarker $\mathbf{K}^{4}$, Arafat $\mathrm{SMG}^{7}$, Ahmad $\mathbf{H}^{1}$, Islam $\mathbf{M M}^{1}$, Ahamed $\mathbf{R}^{8}$ \\ ${ }^{1}$ Department of Geography \& Environment, Jahangirnagar University, Savar, Dhaka, Bangladesh \\ ${ }^{2}$ Communicable Disease Control, Directorate General of Health Services, Mohakhali, Dhaka, \\ Bangladesh \\ Research Article \\ Volume 5 Issue 2 \\ Received Date: May 11, 2021 \\ Published Date: July 28, 2021 \\ DOI: $10.23880 /$ phoa-16000185
}

${ }^{3}$ Mita Clinics, Thana Road, Satkhira Sadar, Satkhira, Bangladesh

${ }^{4}$ Department of Public Health, North South University, Dhaka, Bangladesh

${ }^{5}$ School of Public Health, Independent University Bangladesh, Dhaka, Bangladesh

${ }^{6}$ Climate Change and International Convention, Department of Environment, Agargaon, Dhaka, Bangladesh

${ }^{7}$ Waterkeepers Bangladesh, Blue Planet Initiative, Mohammadpur, Dhaka, Bangladesh

${ }^{8}$ BRAC Institute of Governance and Development, BRAC University, Mohakhali, Dhaka, Bangladesh

*Corresponding author: M Manzurul Hassan, Department of Geography \& Environment, Jahangirnagar University, Savar, Dhaka, Bangladesh, Tel: +8801912151546; Email: manzurulh@gmail.com

\section{Abstract}

Background: Chikungunya virus (CHIKV) transmits to humans by infected mosquitoes of Aedes Aegypti and Aedes Albopictus that has been emerged as a serious public health threat globally. In 2017, Dhaka city of Bangladesh experienced an explosive outbreak of chikungunya, an arthropod-borne virus resulting in more than 13,800 clinically confirmed cases. This paper focuses on the pattern of massive outbreak of chikungunya in Dhaka City as well as the association between different entomological indicators and chikungunya infection.

Methods: The relevant patient information was principally collected from the Institute of Epidemiology, Disease Control and Research (IEDCR) and Dhaka Medical College and Hospital (DMCH), and entomological surveillance data from the Communicable Disease Control (CDC) of Directorate General of Health Services (DGHS) in Dhaka. In addition, surveys were conducted in localities where an outbreak of CHIKV had occurred. A total of 2708 households were visited and a total of 4767 habitats were characterized. Face-to- face interview process was also employed from 436 confirmed patients. The regression analysis, Odds Ratio (OR), and Relative Risk (RR) were used to analyse the risk factors of entomological indicators in making connotation with chikungunya infection. Spatial mapping with proportional symbology and geostatistics were also employed. Results: The overall chikungunya outbreak in 2017 for Dhaka city shows that about 137 per 100,000 population were infected with CHIKV with the average prevalence rate of $1.11 \pm 0.65 \%$ ( $95 \% \mathrm{CI}$ of $0.97-1.25$ ). Some $97.3 \%$ patients had high-grade fever with an average maximum temperature of $103.9 \pm 0.78^{\circ} \mathrm{F}, 88.3 \%$ patients declared for deep and sharp myalgia, and almost all the patients $(98.6 \%)$ reported severe arthralgia in different joints. In addition, the average odds of confirmed chikungunya 
patients in households were found to be 2.43 times (OR: 2.24-2.70 with $95 \% \mathrm{CI}$ and $\mathrm{P}<0.001$ ) and the relative risk was calculated for 2.07 (RR: 1.91-2.24 with 95\% CI and P <0.001) times compared to the infections in negative households in Dhaka City. Moreover, the entomological information shows that out of 4767 habitats, some $12.96 \%$ were positive for Aedes Aegypti larvae and pupae, and positive correlations were found between different entomological indices and chikungunya infections.

Conclusion: Since there is no curative treatment for chikungunya, a proper policy including patient management and Behaviour Change Communication (BCC) as well as strong surveillance strategies should be formulated for the prevention of chikungunya infection as well as to lessen the incidence of mosquito vectors.

Keywords: Chikungunya; Arbovirus; Arthralgia; Myalgia; Aedes Aegypti; Aedes Albopictus; Bangladesh

\section{Introduction}

Chikungunya Virus (CHIKV), an arthropod-borne virus (arbovirus) transmitted to humans by infected mosquitoes of Aedes Aegypti, Aedes Albopictus, and Aedes polynesiensis has recently emerged as a serious public health threat globally [1-7]. CHIKV is an enveloped, spherical, singlestranded positive-sense ribonucleic acid (RNA) virus belongs to the Alphavirus genus of the Togaviridae family $[8,9]$. Chikungunya was first reported in 1952 in Tanzania and was isolated in 1953 from patient serum and mosquitoes $[10,11]$. The term "chikungunya" means as "to become contorted" from its original Makonde (an ethnic group in southeast Tanzania and northern Mozambique) language and describes the stooped appearance, along with joint pain (arthralgia) of the sufferers. It is an etiologic agent of an acute febrile illness that presents symptoms including high fever, headache, myalgia, arthralgia, polyarthralgia, haemorrhage, and rash [12-19]. It is reported that arthralgia is often very debilitating and persists for longer periods, usually lasting for a few days or may be prolonged to weeks or months or years and causes severe pain in older people, as well as in diabetic patients.

Arboviruses are maintained in nature between invertebrate vectors and vertebrate hosts. The mosquitoborne members of the Flaviviridae (e.g., Dengue Virus DENV, West Nile Virus - WNV, Japanese Encephalitis Virus - JEV, and Zika Virus - ZIKV), Togaviridae (e.g., CHIKV), and Bunyaviridae (e.g., Rift Valley Fever Virus - RVFV) account for the majority of human disease cases and present a significant public health threat [2]. In addition, some other mosquito-borne arboviruses have emerged in several specific regions of the world, e.g., St Louis encephalitis virus (SLEV), Murray Valley encephalitis virus (MVEV), Usutu virus (USUV), Spondweni virus (SPOV), and O'nyong nyong virus (ONNV) [20]. Chikungunya still constitutes a major challenge regarding diagnosis, therapeutic and preventive approach, lack of specific antiviral treatment, and the lack of effective immunization [10]. Since its discovery in 1952, CHIKV outbreaks have spread across the globe at an alarming rate causing large numbers of epidemics that have infected millions of people in sub-Saharan Africa, Asia, Europe, the Americas, and recently in Bangladesh.

Chikungunya outbreaks have so far been reported in more than one-third of the countries, mainly in the equatorial and tropical belts in the globe. The Indian subcontinent has experienced several major outbreaks of CHIKV since 2000 and this area is one of the endemic areas of CHIKV [21,22]. In Bangladesh, the most dangerous outbreak of chikungunya in Bangladesh was reported in April-September 2017, when a massive number of positive cases were reported from 23 districts of the country and more than 13,800 clinically confirmed cases were documented in Dhaka city alone and over two million people were at risk of getting infected with the virus [23]. Moreover, reports of sporadic cases of CHIKV infection continued to come in 2013, 2015, and 2016 [24]. The CHIKV from the 2017 outbreak in Dhaka was found to be genetically distinct from the strain found in the previous outbreak, Bangladesh. Continuous surveillance of mosquito-borne diseases like chikungunya is a massive challenge in Bangladesh since most public hospitals lack modern diagnostic facilities for chikungunya. The peak of the outbreak of chikungunya was recorded by the end of May and by the end of July in 2017 [25].

There is a plethora of literature on chikungunya with different aspects all over the world and the relevant literature was designated in a scoping review research of [22] where more than 300 references were cited. Chikungunya is an emerging infection in Bangladesh and there are few papers on chikungunya outbreaks with different themes, e.g., case studies [26], symptoms and situation [27], awareness 
campaign [28], pathogenesis and treatment [29], knowledge about chikungunya [30], limitation of chikungunya diagnosis [25], pattern of outbreak of chikungunya [31], the impacts of chikungunya on economy and quality of life [32], and clinical and hematological aspects [23]. Therefore, the present study aims to explore the spatial pattern of chikungunya outbreak in Dhaka city with the spatial methods as well as risk pattern of chikungunya infection. Moreover, this research investigates the association between different entomological indicators and chikungunya transmission. This paper can contribute to public health and environmental science regarding its health, environmental, and ecological aspects.

\section{Methods}

\section{Research Design}

The research is composed mainly of problem formulation, data collection procedures and manipulation, data analysis and interpretation, and performing geographical analyses with a spatial mapping. The investigation for relevant information from chikungunya patient employed multimethod approach. This strategy provided a mix of both spatial and quantitative data, with the extensive face-to- face interviews through questionnaire survey providing breadth of coverage (Figure 1).

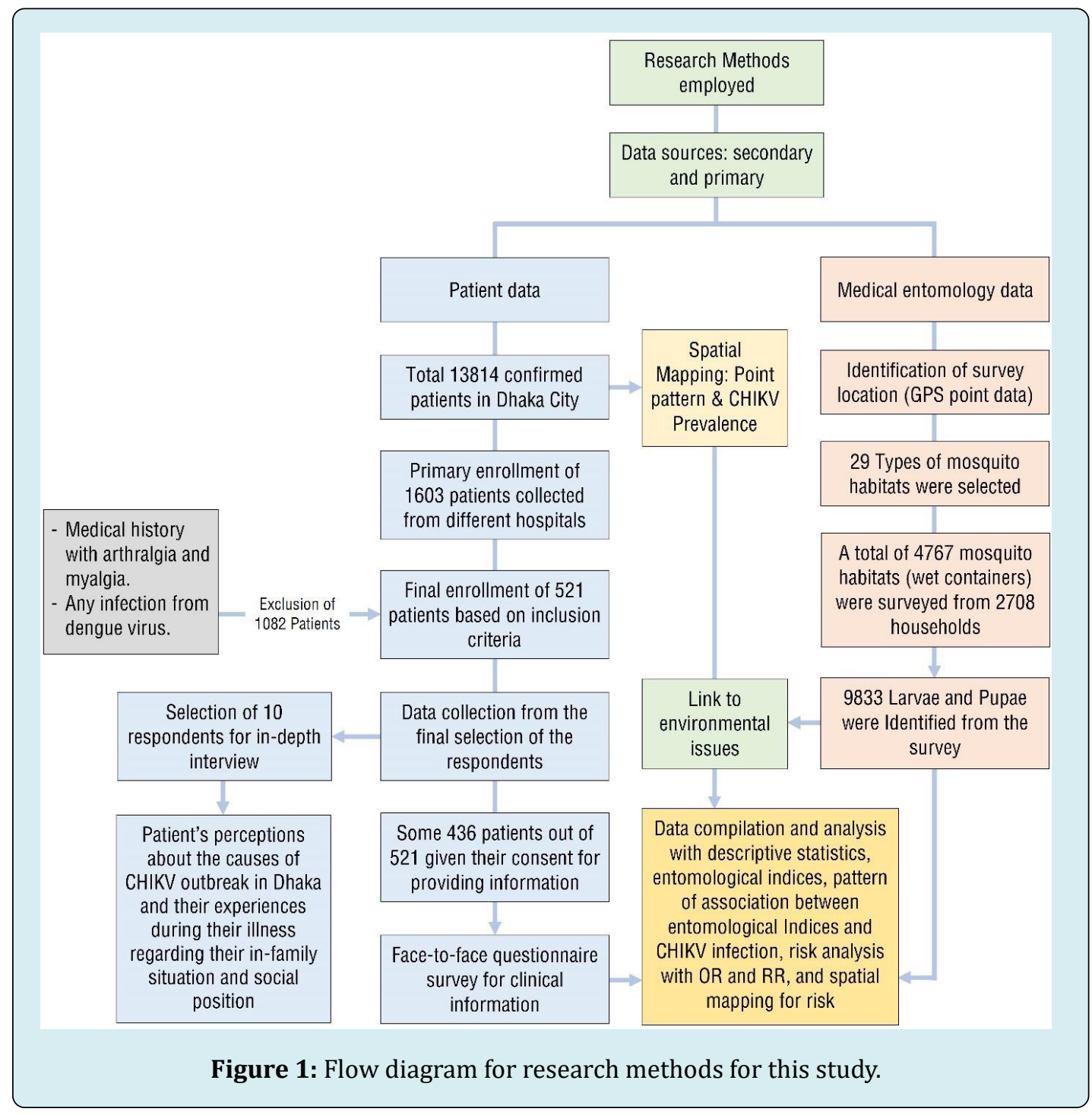

\section{Entomological Surveillance and Vector Control}

The relevant entomological surveillance data used for this research were mainly collected from CDC of DGHS in
Dhaka. The CDC collected relevant entomological surveillance data for preventing CHIKV. Collections of larvae and pupae were made from pools of standing water and wet containers at different houses and its premises from different places in 
Dhaka city, the Capital of Bangladesh (Figure 2). The surveys were conducted in localities where an outbreak of CHIKV had occurred. A multi-staged systematic random sampling procedure was applied for entomological survey. A total of 2708 households were selected for catching larvae-pupae and adult forms of mosquitoes.

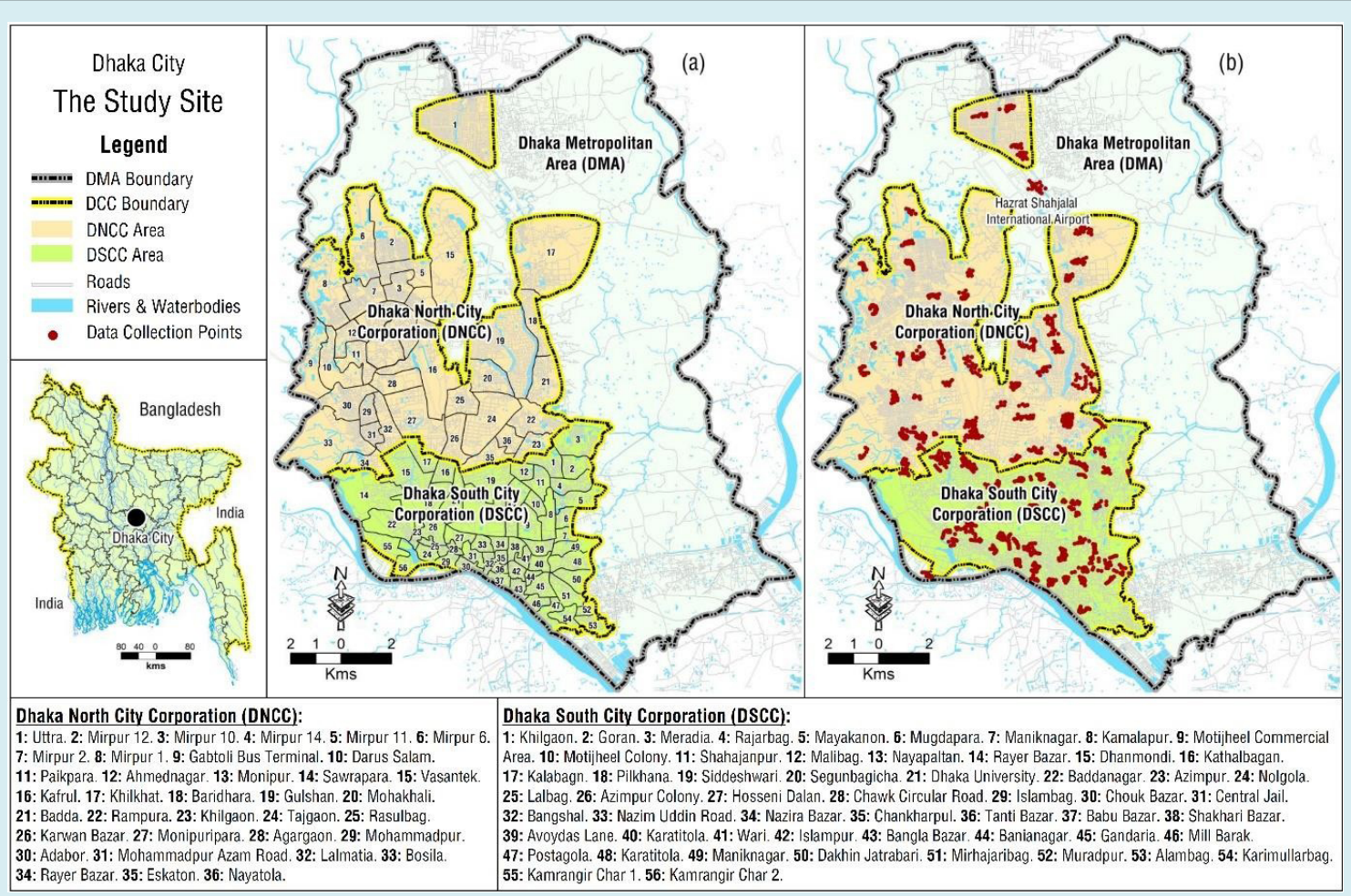

Figure 2: The study site and the study points for larvae and pupae information: (a) Administrative Wards in Dhaka City; and (b) selected points in Dhaka City for entomological survey. It is noted that there are two different administrative units in Dhaka city, and they are Dhaka North City Corporation and Dhaka South City Corporation.

Aedes Aegypti and Aedes Albopictus lay their eggs and larvae subsequently develop in domestic water containers, rainwater-holding objects including a wide variety of discarded materials as well as natural water retaining structures. Some larval habitats were associated with natural water sources, such as ponds, marshy and swamp areas that are permanent or seasonal stagnant water. Several artificial containers such as bottles, cans, plastic and metal pieces, abandoned tires, abandoned refrigerators, and abandoned tubs completed the defined habitat types. Different type of habitats was systematically examined during the field surveys and a total of 4767 habitats yielded mosquito larvae and pupae collections.

In catching of larvae-pupae, the presence of larvae or pupae of Aedes Aegypti was investigated in all containers with water in households. Wet containers inside and outside each selected household were inspected for the presence or absence of Aedes larvae and pupae that were collected by using small dip nets and water pipettes from different habitats. The collected eggs were placed for hatching for species identification. The selected locations of habitats were physically marked on a large scale (RF 1:25000) map of Dhaka City as well as recorded through high-sensitivity Global Positioning System (GPS). The collected GPS data were then synchronized into ArcGIS (version 10x) software for spatial mapping.

\section{Chikungunya Patients and Interview}

The cross-sectional design was employed for this study, and the study was conducted in Dhaka City, the capital of Bangladesh. The patient and entomological surveillance information collected for this study cover almost all the corners of Dhaka city. Relevant CHIKV information, primarily the confirmed patients, was mainly collected from IEDCR and DMCH. In Bangladesh, IEDCR was mainly engaged in monitoring the chikungunya outbreak with Reverse Transcription Polymerase Chain Reaction (RT-PCR) reports. CHIKV cases were defined as patients with fever and additional two or more symptoms such as myalgia, arthralgia, headache, and rash. Some 436 confirmed CHIKV patients 
were interviewed face-to-face at their residences and in some suitable places with semi-structured questionnaire to determine the nature of the disease in terms of clinical symptoms and relevant information regarding the pattern of pain of this disease. Figure 1 shows the flow diagram in selecting the suitable respondents for this study. The fieldwork was conducted in July-September 2018. Before conducting the fieldwork, the selected confirmed patients were asked over telephone for appointments for interview at their residences or any suitable places. After getting their consent, a face-to-face interview process was conducted. In order to fulfill the required sample size considering 95\% Confidence Interval, we approached some 521 residents for their consents about interview, but we received 436 permissions and accordingly we recruited this figure in place of procedural statistical figure of 384 . The respondents were asked a number of questions regarding their medical records, disease symptoms, pains, and recovery as well as their opinions regarding the mitigation and prevention of chikungunya. In addition, formal and informal interviews with different respondents were also made.

\section{Quantitative Analysis}

A bivariate analysis between entomological indices and chikungunya infection was conducted for this study. Linear regression was utilized for analyzing the association between different entomological indices and chikungunya infection. Moreover, risk pattern in terms of OR and RR were used in analyzing the risk pattern of chikungunya infection. The OR is the comparison of the odds from chikungunya infection after exposure to a risk factor with the odds of this CHIKV infection in a control or reference situation. The $\mathrm{OR}$ is estimated as the odds of chikungunya infection in the exposure group divided by the odds of the CHIKV event in the control group. Once more, RR measures the likelihood of chikungunya infection in an exposed group compared to a total non-exposed control group. This RR is estimated as the absolute risk with chikungunya infection divided by the absolute risk in the overall non-exposed control group.

\section{Analysis of Entomological Indices}

Several indicators for vector surveillance were calculated to sustain the control measures and detect any increase in vector density. Surveillance on Aedes Aegypti and Aedes Albopictus density is important in determining factors related to CHIKV transmission. Therefore, larvae and pupae samples were analysed (i.e., House index, Container index, Breteau index, and Pupae index) to prioritize areas for vector control. House index is the percentage of houses infested with larvae and/or pupae (equation (1)); Container index is the percentage of water-holding containers infested with larvae or pupae (equation (2)); Breteau index is the number of positive containers per 100 houses inspected (equation (3)); and Pupae index is the number of pupae per 100 houses inspected (equation (4)). It is noted that positive houses are reported if at least one recipient has one larvae.

\section{Limitation and Bias}

The main limitation of this study is that there is no consideration for age-sex factors since our main objective was to figure out the link between entomological indicators and chikungunya infection. The entomological data were employed to draw up a vector habitat profile and to assess epidemic risk. Several indices, in terms of Breteau index, container index, pupa index, and house index are frequently utilized to assess risk of disease outbreaks and we have deployed these indices for this study. However, there are additional indices e.g., larval index, larvae-pupae index (total number of immature stages per 100 houses), and productivity of a container (number of the third and fourth mosquito larvae stages, L3-4, or pupae in each container type divided by the total number of L3-4 or pupae in all container types) to figure out vector habitat profile as mentioned by Hammond, et al. [33]. These are based on the presence or absence of larvae or pupae as well as their specific numbers. The Breteau, house, and container indices do not consider breeding site productivity but rather consider only the presence or absence of larvae or pupae. It is noted that pupae indices do not consider larvae, which have generally higher density than pupae [33].

\section{Spatial Mapping}

Spatialmappingforvectorinfestation rateswas conducted based on entomological indicators. Each household selected for collecting larvae and pupae was introduced into ArcGIS. Case-patients were plotted into ArcGIS by their address for spatial mapping. Apart from simple point distribution, proportional symbology and choropleth mapping for spatial distribution of patient and spatial pattern of entomological indices were utilized. Moreover, mapping with Ordinary Kriging (OK) estimation was used for regionalised risk pattern with OR and RR against each administrative ward in Dhaka City. The OK interpolation method is used to estimate values when data point values vary or fluctuate around a constant mean value [34-37].

\section{Ethics Statement}

The fieldwork was carried out with the consent of the respondents at their residents. Since the study involves the use of patient medical data, all the data analyzed were anonymized. In addition, no references were made to the 
control individuals. This study was approved by the Biosafety, Biosecurity, and Ethical Committee (BBEC) of Jahangirnagar University, Savar, Dhaka, Bangladesh.

\section{Results}

\section{The Pattern CHIKV Outbreak in Dhaka City}

The overall chikungunya outbreak in 2017 for Dhaka city shows that about 137 per 100,000 populations were infected with CHIKV. The prevalence of CHIKV shows an erratic distribution in Dhaka City (Figure 3). There were total of 13,814 confirmed patients within 92 administrative wards across the Dhaka City. The average prevalence of CHIKV in Dhaka City was calculated for $1.11 \pm 0.65 \%$ (with
95\% CI of 0.97-1.25 and $\mathrm{p}<0.001$ ) having the range between $0.09 \%$ in Maniknagar (Ward 7) to $2.96 \%$ in Mohakhali (Ward 20). Some parts of Dhaka city were identified as the most affected areas for Chikungunya infection. The highest chikungunya infection was recorded in Mohakhali (278, $2.96 \%)$, followed by Lalmatia $(273,2.91 \%)$, Rampura $(271$, $2.89 \%)$, Dhanmondi (268, 2.86\%), Gulshan (265, 2.82\%), Moghbazar (198, 2.11\%), Dhaka University area (192, $2.05 \%)$, Khilgaon (158, 1.68\%), Pilkhana (156, 1.66\%), Meradia $(153,1.63 \%)$, and so on, while the lowest infection was recorded in Maniknagar (8, 0.09\%). Figure 3 shows the distribution pattern of chikungunya infection in Dhaka with different methodological aspects.

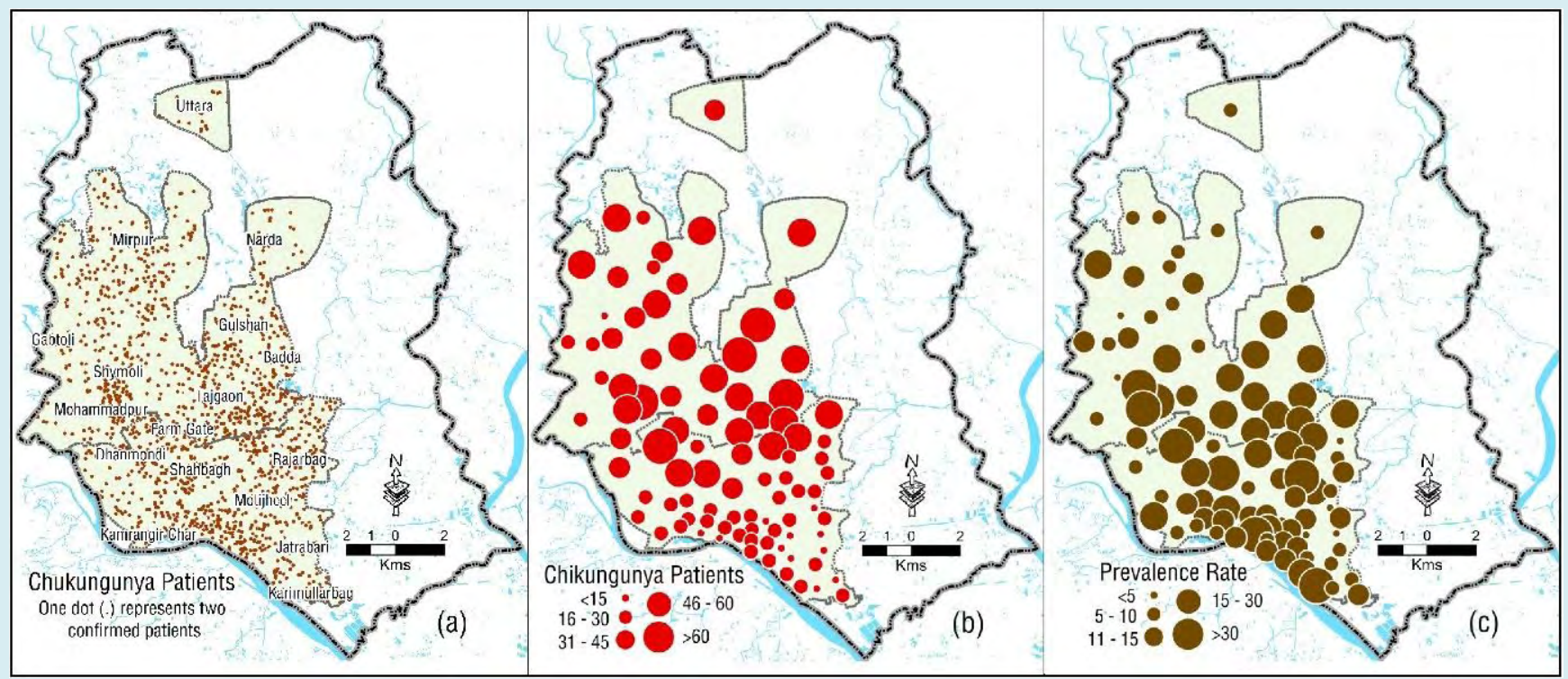

Figure 3: Spatial pattern of CHIKV infection in Dhaka city: (a) point patterns of confirmed CHIKV patients in different administrative wards; (b) CHIKV infection in Dhaka City with proportion symbology; and (c) prevalence of CHIKV in each administrative ward in Dhaka City.

\section{Symptoms Recognition and Clinical Manifestations}

Infection with CHIKV is usually symptomatic and most of the patients infected with CHIKV develop acute symptoms, usually 1 to 5 days after the infective mosquito bite. The first symptoms start abruptly and last for about a week before spontaneous improvement. Our fieldwork shows that chikungunya patients complained more frequently about arthralgia, notably for small joints such as wrists, hands, ankles, and feet. The intensity of joint pains was high in chikungunya patients. Chikungunya patients notified frequently about "burn under feet" and an impaired quality of sleep. They also reported less frequently about "shiver". Amongst the respondents, some 424 (97.3\%) patients had high-grade fever with an average maximum temperature of $103.9 \pm 0.78^{\circ} \mathrm{F}$, some $385(88.3 \%)$ patients declared for deep and sharp myalgia, and almost all the patients (430, 98.6\%) reported severe arthralgia in different joints (Table 1). 


\begin{tabular}{|c|c|c|}
\hline \multirow{2}{*}{ Symptoms } & \multicolumn{2}{|c|}{ Responses (N = 436) } \\
\cline { 2 - 3 } & Frequency & Per Cent \\
\hline High-grade fever (an average temperature of 102-104 ${ }^{\circ} \mathrm{F}$ ) & 424 & 97.3 \\
\hline Severe arthralgia in different joints & 430 & 61.7 \\
\hline Polyarthralgia (polyarticular pain, 5 and more joints) & 269 & 36.9 \\
\hline Oligoarthralgia (oligoarticular pain, 2-4 joints) & 161 & 95.6 \\
\hline Musculoskeletal pain & 417 & 82.6 \\
\hline Joint swelling & 360 & 73.2 \\
\hline Joint stiffness & 319 & 88.3 \\
\hline Deep and sharp myalgia & 385 & 89.6 \\
\hline Skin rash & 391 & 81.7 \\
\hline Itching & 356 & 69.3 \\
\hline Headache & 302 & 74.6 \\
\hline Eye irritation and redness of eye & 325 & 31.9 \\
\hline Throat pain & 139 & 72.3 \\
\hline Dizziness & 315 & 49.1 \\
\hline Despiratory Complaints (e.g., Catarrh-cough) & 214 & 87.6 \\
\hline
\end{tabular}

Table 1: Symptom recognition and clinical manifestations of Chikungunya.

Data Source: Fieldwork, 2018 (multiple responses were considered).

Manifestations of cutaneous symptoms, including skin rash and itching, joint swelling, joint stiffness, headache, and dizziness are common in CHIKV patients musculoskeletal pain (e.g., affects the bones, muscles, ligaments, tendons, and nerves) (417, 95.6\%), skin rash (391, 89.6\%) and joint swelling $(360,82.6 \%)$ were significantly high among the confirmed cases. Amongst the 430 (98.6\%) patients with severe arthralgia, joint pain was mostly recognized with polyarticular ( 5 and more joints) and oligoarticular (2-4 joints). Nearly two-third $(269,61.7 \%)$ of patients had polyarticular pain and more than one-third $(161,36.9 \%)$ of the patients had two to four joints that were affected with pain. Not a single patient mentioned arthralgia specific to a single anatomical site. Arthralgia occurred preferentially in hand joints (fingers and wrists) and in leg joints (knees, ankles and feet). Moreover, shoulder and neck joints are high affected anatomical sites in the chikungunya patients. It is noted that some $76 \%$ of the patients stressed on a symmetric trend of arthralgia. They also mentioned high pain intensity if moves. In addition, the quality of sleep was severely impacted with "very bad" mentioned by a significant number of patients $(382,87.6 \%)$. People affected with CHIKV experienced severe joint pain and the pain level was too high.

Almost all the patients notified severe pain with a mean pain score of $9.3 \pm 0.67$ out of 10 in numerical rating scale.
Some patients in this respect expressed that "those who didn't suffer with chikungunya, they don't recognise the severity of pain of this disease - umm ... severe pain at joints". Some patients said, "pain from chikungunya is probably very close to that of the last breath." Some also mentioned that "chikungunya pain it is intolerable, and it is not comparable to any other type of pain".

\section{Risk Pattern}

Risk pattern from chikungunya infection was calculated with OR and RR methods (Figure 4). A total of 58 administrative wards out of 92 in Dhaka City were recorded with high risk (e.g., OR>1.0). The absolute risk of getting chikungunya infection was found to be 2.43 times (with $95 \%$ CI of 2.24-2.70 and $\mathrm{P}<0.001$ ) with positive households that with relative to negative households in Dhaka City. The highest figure of OR was found in Mirpur-12 (8.87) followed by Banianagar (8.82), Mohammadpur Azam Road (8.72), Islambag (8.51), Chawk Circular Road (8.49), Kawranbazar (8.47), and so on. However, poor odds (e.g., OR<1.0) were found in some 34 administrative wards in Dhaka City (Figure $4 a)$.

$\mathrm{RR}$ is also noticeable in making the association between entomological indicators and chikungunya infection in 
Dhaka City (Figure 4b). The risk of chikungunya infection among the positive households was calculated for 2.07 (with 95\% CI of 1.91-2.24 and P <0.001) times compared to the total negative surveyed households in Dhaka City. This means the positive households are associated with more than doubled risk of getting chikungunya infection that with the negative households in Dhaka City. Furthermore, RR was also calculated in individual wards in Dhaka city. The highest value of RR was found in Mirpur-12 (6.36) followed by Mohammadpur Azam Road (6.29), Islambag (6.19), Chawk Circular Road (6.18), Kawranbazar (6.17) and so on.

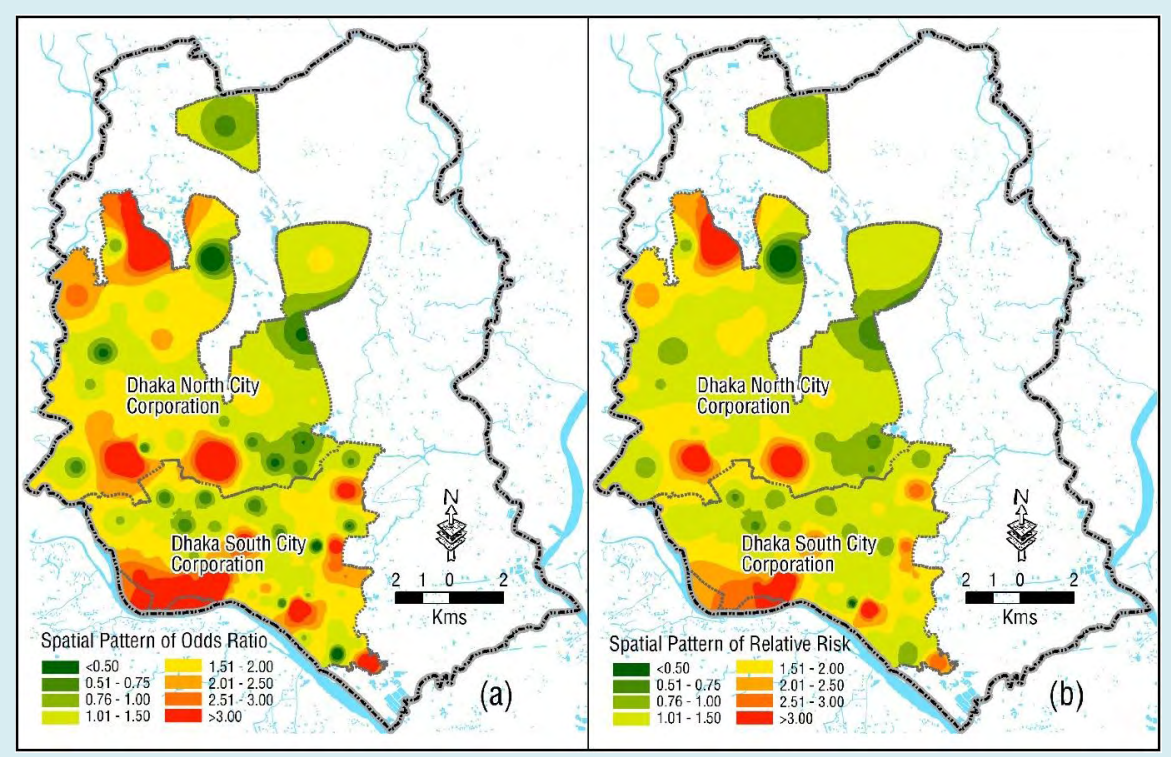

Figure 4: Spatial distribution of CHIKV risks in different administrative areas in Dhaka city: (a) spatial pattern of Odds Ratio, and (b) spatial pattern of Relative Risk

\section{Entomological Indicators and Chikungunya Infection}

The entomological indicators reveal the root-cause of chikungunya infection in Dhaka City. Our investigation shows that some $618(12.9 \%)$ positive containers were identified from a total of 4767 surveyed wet containers. The maximum positive containers were identified as plastic bucket $(15.86 \%)$ followed by unused tires $(14.40 \%)$, basement flooded floor $(12.14 \%)$, clay pot $(8.74 \%)$, money plant tub
(6.31\%), metal drum (5.66\%) and so on. In addition, a total of 9833 larvae and pupae were identified from these 618 positive containers. In addition, the highest number of larvae and pupae were identified from different mosquito habitats. The most suitable habitats were found in unused tires (1492, 15.17\%), followed by plastic buckets $(1167,11.87 \%)$, metal drum $(1140,11.59 \%)$, basement flooded floor $(1119$, $11.38 \%)$, clay pot $(859,8.74 \%)$, money plant tub (669, $6.80 \%)$, tin/metal can $(499,5.07 \%)$, and so on (Table 2 ).

\begin{tabular}{|c|c|c|c|c|c|}
\hline Container Types & \multirow{2}{*}{ Visited Wet Containers } & \multicolumn{2}{|c|}{ Positive Containers } & \multicolumn{2}{c|}{ Identified Larvae \& Pupae } \\
\cline { 3 - 5 } (Mosquito habitats) & & Frequency & Net Percent & Frequency & Net Percent \\
\hline Water tank & 174 & 18 & 2.91 & 273 & 2.78 \\
\hline Cement pot & 22 & 3 & 0.49 & 66 & 0.67 \\
\hline Clay pot & 340 & 54 & 8.74 & 859 & 8.74 \\
\hline Ceramic pot & 26 & 10 & 1.62 & 109 & 1.11 \\
\hline Aluminum pot & 23 & 6 & 0.97 & 188 & 1.91 \\
\hline Glass bottle & 42 & 1 & 0.16 & 21 & 0.21 \\
\hline Plastic bottle & 206 & 15 & 2.43 & 135 & 1.37 \\
\hline Plastic mug & 156 & 26 & 4.21 & 285 & 2.9 \\
\hline Earthen jar (Motka) & 52 & 9 & 1.46 & 84 & 0.85 \\
\hline Tin/metal can & 113 & 24 & 3.88 & 499 & 5.07 \\
\hline
\end{tabular}




\begin{tabular}{|c|c|c|c|c|c|}
\hline Metal pan & 27 & 4 & 0.65 & 72 & 0.73 \\
\hline Metal bucket & 63 & 6 & 0.97 & 13 & 0.13 \\
\hline Plastic bucket & 712 & 98 & 15.86 & 1167 & 11.87 \\
\hline Plastic bags & 56 & 5 & 0.81 & 66 & 0.67 \\
\hline Animal bowl & 24 & 5 & 0.81 & 154 & 1.57 \\
\hline Flag stand hole & 24 & 4 & 0.65 & 25 & 0.25 \\
\hline Metal drum & 335 & 35 & 5.66 & 1140 & 11.59 \\
\hline Plastic drum (sealable) & 176 & 25 & 4.05 & 332 & 3.38 \\
\hline Money plants tub & 914 & 39 & 6.31 & 669 & 6.8 \\
\hline Flower tub and tray & 310 & 16 & 2.59 & 158 & 1.61 \\
\hline Tires & 256 & 89 & 14.4 & 1492 & 15.17 \\
\hline Cement mixer & 3 & 2 & 0.32 & 70 & 0.71 \\
\hline Discarded food container & 163 & 15 & 2.43 & 249 & 2.53 \\
\hline Plastic sheet or tarp & 32 & 4 & 0.65 & 26 & 0.26 \\
\hline Flooded floor (basement) & 251 & 75 & 12.14 & 1119 & 11.38 \\
\hline Water Jar & 246 & 18 & 2.91 & 320 & 3.25 \\
\hline Tree hole & 6 & 4 & 0.65 & 30 & 0.31 \\
\hline Battery shell & 7 & 1 & 0.16 & 130 & 1.32 \\
\hline Discarded toilet parts & 8 & 7 & 1.13 & 82 & 0.83 \\
\hline Total & 4767 & 618 & 100 & 9833 & 100 \\
\hline
\end{tabular}

Table 2: Selected habitat types for analyzing the presence of mosquito larvae and pupae in the surveyed areas of Dhaka City.

The average House index was counted for $26.15 \pm 14.67$ (with 95\% CI of 23.09-29.20 and $\mathrm{P}<0.001$ ) and the maximum House index was recorded for Lalmatia (65.71) followed by Ahmednagar (55.0), Nayatola (54.28), and Kathalbagan (51.42). It is noted that the vector infestation level was based on House index, where the risk classification is defined as: low $(<2)$, moderate (2-4) and high ( $>4)$. The distribution pattern of House index shows that about $95 \%$ of the administrative wards are within the category of high risk zone. The typical Container index was found to be high in some parts of the city and the index was calculated for $20.15 \pm 17.72$ (with 95\% CI of 16.46-23.85 and $\mathrm{p}<0.001$ ) where the maximum Container index was logged for 100 in both Kawran Bazar and Mayakanon followed by Bosila (75.0), Pilkhana (58.33),
Alambag (50.0), and so on. The Breteau index was found to be high in most of the areas of the city and the average Breteau index was considered for $34.88 \pm 20.97$ (with $95 \%$ CI of 30.51 39.25 and $\mathrm{p}<0.001)$. The ranking of Breteau index was found in Bosila (90.0) followed by Ahmednagar (80.0), Siddeshwari (77.33), Dhaka University area (77.14), Mayakanon (75.0), and so on. Finally, the pPupae index was also found to be extreme in the city and the average Pupae index was counted for $28.85 \pm 53.74$ (with $95 \%$ CI of $17.66-40.04$ and $p<0.001$ ) having the maximum value was recorded to Mayakanon (325.0) followed by Shakhari Bazar (250.0), Dhanmondi (158.75), Rasulbag (125.0), Shajahanpur (122.86), and so on (Table 3).

\begin{tabular}{|c|c|c|c|c|c|}
\hline Entomological Indicators & Mean & Std. Deviation & Std. Error & 95\% CI & $\boldsymbol{P}$-value \\
\hline House Index & 26.15 & 14.67 & 1.54 & $23.09-29.20$ & $<0.001$ \\
\hline Container Index & 20.15 & 17.72 & 1.86 & $16.46-23.85$ & $<0.001$ \\
\hline Breteau Index & 34.88 & 20.97 & 2.19 & $30.51-39.25$ & $<0.001$ \\
\hline Pupae Index & 28.85 & 53.74 & 5.63 & $17.66-40.04$ & $<0.001$ \\
\hline
\end{tabular}

Table 3: Distribution of entomological indicators of CHIKV in Dhaka City.

Note: Implies statistically significant at $1 \%$ level.

The density of Aedes Aegypti was high in the study site. All the entomological indicators calculated for this study were found to be inconsistent and irregular in Dhaka city, but high concentrations were recognized with different magnitudes in different administrative wards (Figure 5). The overall pattern of entomological indices was very uneven, the figures of each index has been depicted different scenarios of mosquito environment of Aedes Aegypti and their breeding sites. 


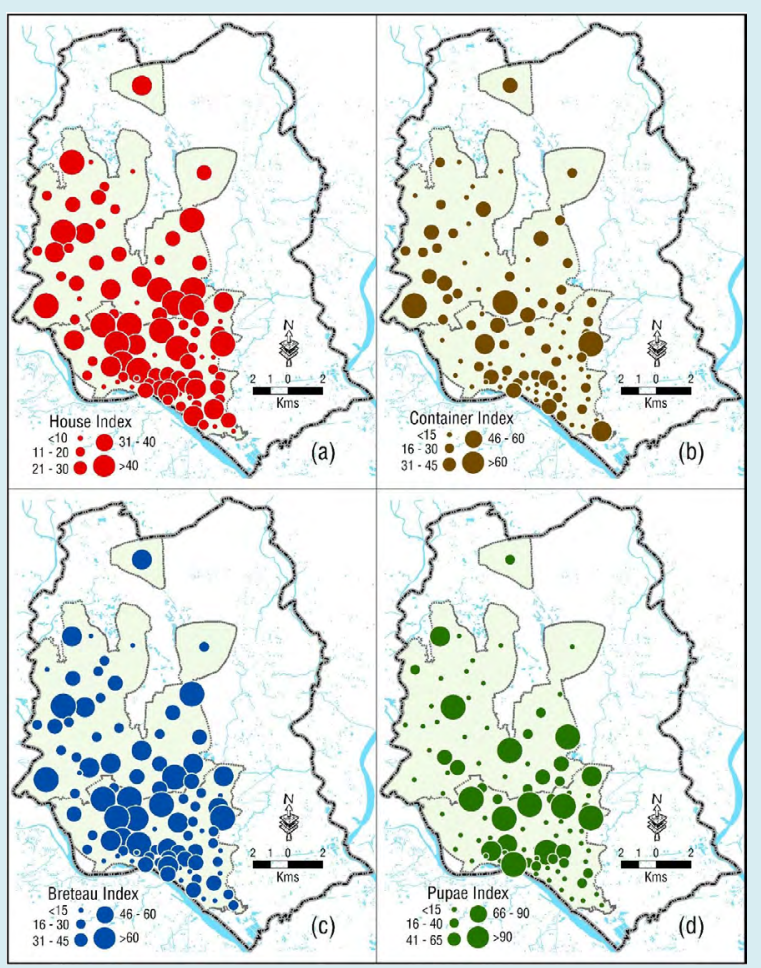

Figure 5: Spatial pattern of entomological indices for CHIKV infection in different areas in Dhaka City: (a) House index; (b) Container index; (c) Breteau index; and (d) Pupae index.

There are diversified relationships between different entomological indicators and chikungunya infection. High correlation between House index and chikungunya infection was noted from our fieldwork and the association was measured for +0.76 . Likewise, high association $(+0.69)$ was found between Breteau index and chikungunya infection. A moderate association $(+0.57)$ was measured for Pupae index and a low association $(+0.38)$ was restrained between Container index and chikungunya infection (Figure 6). A significant number of the surveyed houses, in and around, in the study sites were with different types of wet containers which served as breeding sites and the main sources of Aedes Aegypti mosquitoes. This is supported by different levels of entomological indicators which are indicative for high Aedes Aegypti densities, appear to be responsible for widespread transmission of chikungunya infection in Dhaka City.

The transmission of CHIKV shows sporadic epidemiological pattern in Dhaka City. It was observed from epidemiological data that viral spread was largely driven by transmissions at distances not much further away than neighbouring households in Dhaka City. It was found from our fieldwork that houses with suitable environment (e.g., water containers, plastic bottles, flower tub, and so on) for Aedes mosquito vectors showed multiple chikungunya patients in a household, while incompatible in-house environment for Aedes mosquito vectors shows no chikungunya infection or only one infected person in a household. Chikungunya outbreak in Dhaka during 2017 was thought to be the caused by climate suitability, and very unusual rainfalls during the winter and wet seasons during that year. Water logging in Dhaka city during the monsoon in every year is another cause of high mosquito density. Moreover, unhygienic household environment in different parts of the city and clogged drainage system with solid waste or with washed-in debris in Dhaka City are responsible for Aedes mosquito vectors.

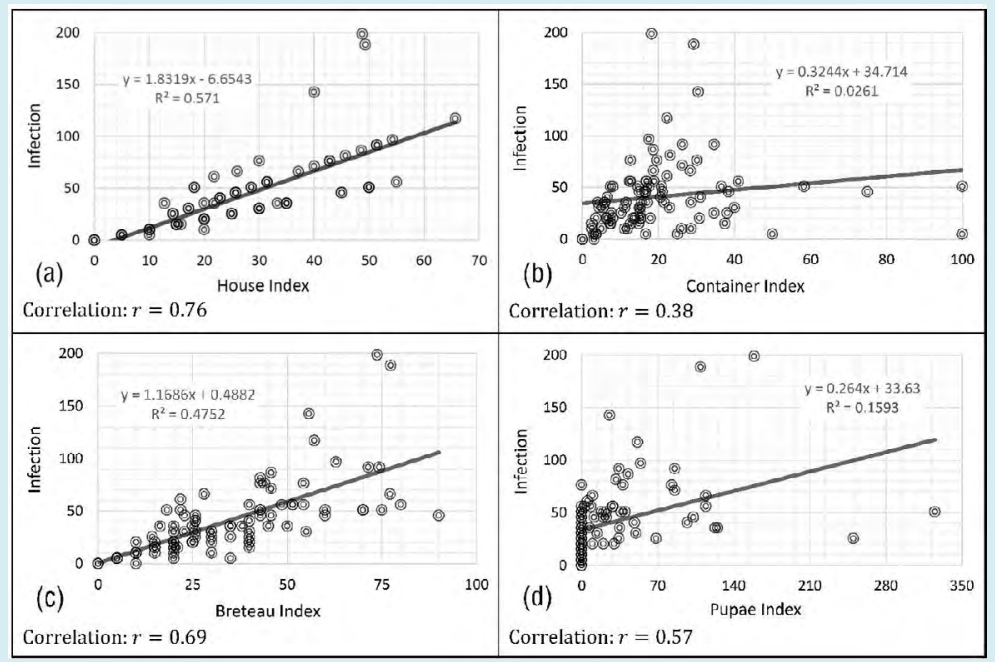

Figure 6: Association between entomological indices and chikungunya infection: (a) House index and chikungunya infection; (b) Breteau index and chikungunya infection; (c) Pupae index and chikungunya infection; and (d) Container index and chikungunya infection. 


\section{Discussion}

This study examines the spatial distribution of entomological indicators of larvae-pupae and adults for mosquitoes. Simultaneously, we analyzed the spatial distribution of CHIKV prevalence and its association with entomological indicators. Infected mosquitoes are responsible for CHIKV transmission through horizontal and vertical transmission. CHIKV is mainly transmitted from humans to mosquitoes to humans during outbreaks [38]. CHIKV from the 2017 outbreak strains in Dhaka constitute a new cluster within the Indian Ocean Lineage (IOL), associated with E1 sequence diversity, suggesting that they are novel variants within the East-Central-South African (ECSA) (E1-A226V) genotype [39]. In addition, E2- L210Q mutation has been found to cause a dramatic increase in the infectivity of CHIKV [9].

Apart from a massive chikungunya outbreak in Dhaka by 2017 , four small and localised outbreaks were recorded during 2008-2012 in Bangladesh. The first recognized Chikungunya outbreak in Bangladesh was identified in December 2008 in Rajshahi and Chapai Nawabganj districts with a total of 39 cases $[26,31,40,41]$. These 39 patients were from a community of potters, and larvae of Aedes Albopictus were found in water contained in numerous pots kept in and around houses of this potters' community. The second outbreak was recognized in Shathiya upazila (sub-district) of Pabna district in 2009. Thereafter, serologic evidence confirmed occurrence of outbreaks and sporadic cases reported in rural areas of Dhaka. In the late October 2011, an outbreak of CHIKV with severe joint pain was reported at Dohar in Dhaka district. In analyzing blood samples from 245 suspected cases, some 196 (80\%) had confirmed CHIKV [31] and Aedes Albopictus was responsible for transmission during this outbreak [42]. In 2012, another outbreak of CHIKV was reported at Palpara village in Tangail district. A total of 175 CHIKV cases (48.1\%) was confirmed using serology test out of 364 individuals reported having suffered from symptoms consistent with chikungunya infection $[42,43]$. By the end of 2012, a total of 600 suspected patients from Sutrapur,

Dhanmondhi, Motijheel and Mohakhali areas in Dhaka City were tested for chikungunya and found 198 confirmed CHIKV patients [41]. It was indicated that people at Mandera area in Kenya usually store water in underground tanks in all homesteads which serve as permanent breeding sites and continuous source of Aedes Aegypti mosquitoes. This is supported by high entomological indicators (e.g., House index by $14.5 \%$; Container index by $41.9 \%$; and Breteau index by $17.1 \%$ ) which are useful in indicating the breeding sites for Aedes Aegypti [44]. These high indices appear to be responsible for widespread transmission of chikungunya in Kenya. Our study also shows high entomological indices in different parts of the city and it shows statistically positive correlations between different indices and infection. However, the study observed that the clusters of CHIKV prevalence are not high corresponding with the distribution of entomological indicators in all the areas of Dhaka City although there are positive correlations between entomological indicators and chikungunya infection (Figure 6).

Both Aedes Aegypti and Aedes Albopictus are forest species that exploit small, natural containers of water (e.g., holes in trees, fruit husks, and plant axils) for their aquatic, developmental stages [45]. They adapt to the urban environment in habiting a variety of artificial water containers. Aedes Aegypti is highly anthropophilic and is primarily endophilic [46]. Aedes Albopictus is generally frequent in rural environment and it maintains of the CHIKV sylvatic cycle. It feeds on animals and it mainly breeds in outdoor storage and discarded containers. Although Aedes Aegypti has been the principal vector, but Aedes Albopictus was also a competent vector of CHIKV outbreaks in Dhaka. Rozilawati, et al. [47] investigated positive association between wet containers and Aedes Albopictus. They inspected a total of 2,814 artificial containers in localities in the State of Kelantan, Malaysia and they found that 97 containers were positive. The most abundant containers available were plastic containers (41\%), followed by pail (12\%), tires $(11 \%)$ and water containers (8\%). However, none were positive with Aedes Aegypti. However, study shows that 618 mosquito habitats out of selected wet containers of 4767 were positive with Aedes Aegypti.

High fever and severe arthralgia can be regarded as the cardinal hallmark of the chikungunya 2017 outbreak in Bangladesh. Our study found $97.3 \%$ patients had high-grade fever with an average maximum temperature of $103.9 \pm 0.78^{\circ} \mathrm{F}$, some $88.3 \%$ patients declared for deep and sharp myalgia, and almost all the patients $98.6 \%$ reported severe arthralgia in different joints. The frequency of incapacitating pain in the acute phase involving certain peripheral joints was found to be comparable with a number of literature (e.g., 23,48,49); nevertheless, the results are contrasted with different literature [48-51]. In case of entomological indicators, visiting a total of 2708 households and a total of 4767 characterized habitats, we found more than one-seventh (12.96\%) of analysed containers were positive for Aedes Aegypti larvae- pupae, while Hammond, et al. [33] analysed a total of 7607 containers in 2003, of which $11 \%$ were positive for larvae and 4\% for pupae for Aedes Aegypti. In addition to barrels, they identified potted plants and superficial water on tarps and in puddles as highly productive sites.

Vector control is vital to restrain transmission and prevent outbreaks. Historical trend provides an indication 
of controlling of arboviral vector for DENV, CHIKV, and YFV. In the early 20th century, "clean-up campaigns" were conducted for systematic elimination of breeding sites about a remarkable reduction in urban YFV [45]. This "source reduction" was highly effective with the use of DDT in the 1950s. This "per- focal treatment" was effective and by the early 1960s, the species was declared absent from many countries. Unfortunately, for a number of reasons, Aedes Aegypti remerged, quickly followed by the reappearance of epidemic DENV [52]. Since the "source reduction" campaign has not been achieved significantly, the sustained control of CHIKV or DENV was remained impossible. Apart from the use of biological agents, "source reduction" through the application of toxic and chemical products to aquatic stages is a viable and immediate vector control strategy. Historically, the control of adult mosquito populations was based on the use of insecticides alone or insecticide-treated physical barriers (e.g., bed nets and curtains) [45]. However, the widespread use of insecticides has led to increased resistance in vector populations, causing the need for the development of alternative strategies [53].

\section{Conclusion}

The proximity of mosquito vector breeding sites to human habitation is a significant risk factor for CHIKV transmission. Given the pattern of spatiotemporal dynamics of chikungunya outbreak, it is vital to formulate entomological surveillance system and effective management approach of vector control. The study shows that the spatial pattern of disease depends on the vector distribution with different degrees, and this could detect on a scale of neighbourhoods. It is noted that apart from environmental concerns, climate variables seem to determine the spread of the epidemic in infestation areas. Therefore, prevention of transmission of chikungunya can be possible with maintaining vector infestation at low levels. Since there is no commercial vaccine or effective treatments for CHIKV, vector control and prevention remain the best strategies to minimize the brunt of CHIKV transmission. Before the outbreak of chikungunya in Dhaka in 2017, it was almost unknown in Bangladesh and most of the cases remain undiagnosed or sometimes misdiagnosed due to lack of awareness and diagnostic facilities of chikungunya. Moreover, prevalence of dengue fever, another arthropodborne disease is common in Bangladesh, lead to confusion due to them having overlapping symptoms. Productive wet containers are needed to detect chikungunya and dengue viruses continuously and timely through sustained entomological surveillance and vector control programs to prevent possible outbreaks.

The prevention relies heavily on reducing the number of natural and artificial water-filled container habitats that support breeding of the mosquitoes. An integrated vector management approach is necessary for mosquito control. Several measures in this connection can be significant and they are: (a) anti-larval measures, i.e., environmental control, chemical control, and biological control; (b) antiadult measures, i.e., space sprays, residual sprays, and genetic control; (c) protection against mosquito bites, i.e., mosquito nets, screening, and repellents; and (d) legislative control, i.e., Civic Laws. Environmental factors in terms of basic sanitation, proper waste management and disposal, and proper sewage system as part of alternative strategies that could indirectly contribute to eliminate breeding sites of mosquitoes.

Persistent Behaviour Change Communication (BCC) as a key strategy for CHIKV control. Its aim is to use education campaigns to encourage the reduction or elimination of infested containers. Special campaigns may be carried out through mass media, radio and television, especially using local cable networks. Moreover, a continuous national integrated "entomological surveillance system" should be established to continuous monitor the effectiveness of the control measure to reduce arboviral transmission with continued survey in pre-monsoon, monsoon, and postmonsoon.

\section{References}

1. Aly MM, Ali S, Muianga AF, Monteiro V, Gallego JG, et al. (2017) Severe Chikungunya infection in Northern Mozambique: a case report. BMC 10(1): 88.

2. Brackney DE (2017) Implications of autophagy on arbovirus infection of mosquitoes. Current Opinion in Insect Science 22: 1-6.

3. Cerbino-Neto J, Mesquita EC, Amancio RT, Brasil PE (2020) Events preceding death among chikungunya virus infected patients: a systematic review. Revista da Sociedade Brasileira de Medicina Tropical 53: e04312019.

4. Chirathaworn C, Chansaenroj J, Poovorawan Y (2020) Cytokines and Chemokines in Chikungunya Virus Infection: Protection or Induction of Pathology. Pathogens 9(6): 415.

5. Kumar R, Shrivastava T, Samal S, Ahmed S, Parray HA (2020) Antibody-based therapeutic interventions: possible strategy to counter chikungunya viral infection. Appl Microbiol Biotechnol 104: 3209-3228.

6. Mahdy AA, Jamal M, Kinoshita H, Hossan T (2018) Chikungunya Virus Outbreak - a Threat to Global Public Health including Bangladesh. Bangladesh Journal of Medical Science 17(2): 183-184. 
7. Wang Y, Wang X, Liu X, Ren R, Zhou L, et al. (2019) Epidemiology of Imported Infectious Diseases, China, 2005-2016. Emerg Infect Dis 25(1): 33-41.

8. Suhana O, Nazni WA, Apandi Y, Farah H, Lee HL, et al. (2019) Insight into the origin of chikungunya virus in Malaysian non-human primates via sequence analysis. Heliyon 5(12): e02682.

9. Wahid B, Ali A, Rafique S, Idrees M (2017) Global expansion of chikungunya virus: mapping the 64- year history. Int J Infect Dis 58: 69-76.

10. Martins MM, Prata-Barbosa A, Da Cunha AJ (2020) Arboviral diseases in pediatrics. J Pediatr (Rio J) 96(S1): 2-11.

11. Robinson MC (1955) An epidemic of virus disease in Southern Province, Tanganyika territory in 1952-1953. Trans R Soc Trop Med Hyg 49(1): 28-32.

12. Bozza FA, Moreira-Soto A, Rockstroh A, Fischer C, Nascimento AD, et al. (2019) Differential shedding and antibody kinetics of Zika and Chikungunya viruses, Brazil. Emerg Infect Dis 25(2): 311-315.

13. Cunha MS, Costa PA, Correa IA, de Souza MR, Calil PT, et al. (2020) Chikungunya Virus: An Emergent Arbovirus to the South American Continent and a Continuous Threat to the World. Front Microbiol 11: 1297.

14. Gordon A, Gresh L, Ojeda S, Chowell G, Gonzalez K, et al. (2018) Differences in transmission and disease severity between 2 successive waves of Chikungunya. Clin Infect Dis 67(11): 1760-1767.

15. Halstead SB (2018) Chikungunya and Zika Disease. In: Higgs S, Vanlandingham DL, Powers AM (Eds.), Chikungunya and Zika Viruses. Academic Press, United States, pp: 69-85.

16. Jones R, Kulkarni MA, Davidson TM, RADAM-LAC Research Team, Talbot B (2020) Arbovirus vectors of epidemiological concern in the Americas: A scoping review of entomological studies on Zika, dengue and chikungunya virus vectors. Plos ONE 15(2): e0220753.

17. KhongwichitS, ChansaenrojJ, Thongmee T,Benjamanukul S, Wanlapakorn N, et al. (2021) Large-scale outbreak of Chikungunya virus infection in Thailand, 2018-2019. PLoS ONE 16(3): e0247314.

18. Martinez JD, Cardenas GJA, Cuellar BA (2019) Going viral 2019: Zika, chikungunya, and dengue. Dermatol Clin 37(1): 95-105.

19. Nyamwaya DK, Otiende M, Omuoyo DO, Githinji G,
Karanja HK, et al. (2021) Endemic chikungunya fever in Kenyan children: a prospective cohort study. BMC Infectious Diseases 21: 186.

20. Gould E, Pettersson J, Higgs S, Charrel R, Lamballerie DX (2017) Emerging arboviruses: why today? One Health 4: 1-3.

21. Caputo B, Russo G, Manica M, Vairo F, Poletti P, et al. (2020) A comparative analysis of the 2007 and 2017 Italian chikungunya outbreaks and implication for public health response. PLoS Negl Trop Dis 14(6): e0008159.

22. Mascarenhas M, Garasia S, Berthiaume P, Corrin T, Greig J (2018) A scoping review of published literature on chikungunya virus. PloS ONE 13(11).

23. Anwar S, Mourosi JT, Khan MF, Ullah MO, Vanakker OM, et al. (2020) Chikungunya outbreak in Bangladesh (2017) Clinical and hematological findings. PLoS Neglected Tropical Disease 14(2): e0007466.

24. Alam MT (2018) Chikungunya: Bangladesh perspective. Faridpur Medical College Journal 13(1): 1.

25. Kabir I, Dhimal M, Muller R, Banik S, Haque U (2017) The 2017 Dhaka chikungunya outbreak. The Lancet Infectious Disease 17(11): 1118.

26. Rahim MA, Uddin KN (2017) Chikungunya: an emerging viral infection with varied clinical presentations in Bangladesh: Reports of seven cases. BMC Research Notes 10(1): 410.

27. Faiz MA, Alamgir AS (2017) Chikungunya Outbreak in Dhaka: Lessons for Bangladesh. Journal of Bangladesh College of Physicians and Surgeons 35(3): 108-109.

28. Pervin M (2017) Emerging Threat of Chikungunya: Bangladesh Perspective. Journal of Dhaka Medical College 26(1): 1-2.

29. Rahman S, Suchana S, Rashid S, Pave O (2017) A review article on chikungunya virus. World Journal of Pharmaceutical Research 6(13): 100-107.

30. Mustari S (2018) Knowledge about Chikungunya: A Case with Private University Students' of Dhaka, Bangladesh. European Journal of Social Sciences 57(1): 70-79.

31. Khatun S, Chakraborty A, Rahman M, Banu NN, Rahman MM (2015) An outbreak of chikungunya in rural Bangladesh, 2011. PLoS Negl Trop Dis 9(7).

32. Hossain MS, Hasan MM, Islam MS, Islam S, Mozaffor M, et al. (2018) Chikungunya outbreak (2017) in Bangladesh: Clinical profile, economic impact and quality of life 
during the acute phase of the disease. PLoS Negl Trop Dis 12(6): e0006561.

33. Hammond SN, Gordon AL, del C Lugo E, Moreno G, Kuan GM (2007) Characterization of Aedes Aegypti (Diptera: Culcidae) production sites in Urban Nicaragua. J Med Entomology 44(5): 851-860.

34. Hassan MM (2005) Spatial risk pattern for arsenic contamination: mapping with inverse distance weighting (IDW) method. International Society for Photogrammetry and Remote Sensing Proceedings. The $4^{\text {th }}$ Workshop on Dynamic \& Multi-dimensional GIS. Pontypridd, Wales, UK. 5-8 September, XXXVI, Part 2/ W29, pp: 52-56.

35. Hassan MM (2018) Arsenic in groundwater: poisoning and risk assessment. CRC Press, pp: 377.

36. Hassan MM, Ahamed R (2017) Arsenic-safe aquifers in coastal Bangladesh: an investigation with ordinary kriging estimation. International Archives of the Photogrammetry, Remote Sensing \& Spatial Information Sciences XLII-4/W5: 97-105.

37. Isaaks EH, Srivastava RM (1989) An introduction to applied geostatistics. Oxford University Press, New York, USA.

38. Pastula DM, Smith DE, Beckham JD, Tyler KL (2016) Four emerging arboviral diseases in North America: Jamestown Canyon, Powassan, chikungunya, and Zika virus diseases. J Neurovirol 22(3): 257-260.

39. Melan A, Aung MS, Khanam F, Paul SK, Riaz BK, et al. (2018) Molecular characterization of chikungunya virus causing the 2017 outbreak in Dhaka, Bangladesh. New Microbes New Infect 24: 14-16.

40. Chowdhury FI, Kabir A, Das A, Mukerrama SM, Masud S (2012) Chikungunya Fever: An Emerging Threat to Bangladesh. Journal of Medicine 13(1): 60-64.

41. Herriman R (2014) Bangladesh health officials report chikungunya in Dhaka. IEDCR.

42. Amin MR, Rahman MM, Islam QT (2017) Chikungunya. Journal of Medicine 18(2): 92-108.

43. Salje H, Lessler J, Paul KK, Azman AS, Rahman MW, et al.
(2016) How social structures, space, and behaviors shape the spread of infectious diseases using chikungunya as a case study. Proc Natl Acad Sci U S A 113(47): 1342013425.

44. Konongoi SL, Nyunja A, Ofula V, Owaka S, Koka H, et al. (2018) Human and entomologic investigations of chikungunya outbreak in Mandera, Northeastern Kenya, 2016. PloS ONE 13(10): e0205058.

45. Diallo M, Dia I, Diallo D, Diagne CT, Ba Y, et al. (2016) Perspectives and Challenges in Entomological Risk Assessment and Vector Control of Chikungunya. J Infect Dis 214(5): S459-S465.

46. Lounibos LP (2002) Invasions by insect vectors of human disease. Annu Rev Entomology 47: 233-266.

47. Rozilawati H, Faudzi AY, Rahidah AAS, Azlina AHN, Abdullah AG, et al. (2011) Entomological study of chikungunya infections in the State of Kelantan, Malaysia. Indian J Med Res 133(6): 670-673.

48. Queyriaux B, Simon F, Grandadam M, Michel R, Tolou $\mathrm{H}$, et al. (2008) Clinical burden of chikungunya virus infection. Lancet Infect Dis 8(1): 2-3.

49. Staikowsky F, Le Roux K, Schuffenecker I, Laurent P, Grivard P, et al. (2008) Retrospective survey of Chikungunya disease in Reunion Island hospital staff. Epidemiol Infect 136(2): 196-206.

50. van Genderen FT, Krishnadath I, Sno R, Grunberg MG, Zijlmans W, et al. (2016) First chikungunya outbreak in Suriname; clinical and epidemiological features. PLoS neglected tropical diseases 10(4).

51. Vijayakumar KP, Anish TS, George B, Lawrence T, Muthukkutty SC, et al. (2011) Clinical profile of chikungunya patients during the epidemic of 2007 in Kerala, India. J Glob Infect Dis 3(3): 221-226.

52. Soper FL (1963) The elimination of urban yellow fever in the Americas through the eradication of Aedes Aegypti. Am J Public Health Nations Health 53(1): 7-16.

53. Liu N (2015) Insecticide resistance in mosquitoes: impact, mechanisms, and research directions. Annu Rev Entomology 60: 537-559. 